\begin{tabular}{|c|c|}
\hline Title & Transmitting pro-environmental behaviours to the next generation : A comparison between Germany and Japan \\
\hline Author(s) & A ndo, Kaori; Y orifuji, Kay o; OHNUMA, Susumu; Matthies, Ellen; Kanbara, A yumi \\
\hline Citation & $\begin{array}{l}\text { A sian Journal of Social Psychology, 18(2), } 134144 \\
\text { https://doi.org/10.1111/ajsp.12078 }\end{array}$ \\
\hline Issue Date & $2015-06$ \\
\hline Doc URL & http:/hdl.handle.net/2115/62345 \\
\hline Rights & $\begin{array}{l}\text { A uthor Posting. } \odot \text { The A uthors } 2015 \text { This is the author's version of the work. It is posted here for personal use, not for } \\
\text { redistribution. The definitive version was published in A sian Journal of Social Psychology, V olume 18, Issue 2, pages } \\
134-144 \text {, June 2015. http://dx.doi.org/10.1111/aj sp.12078 }\end{array}$ \\
\hline Type & article (author version) \\
\hline File Information & Elementary school survey_140401ando_final.pdf \\
\hline
\end{tabular}

Instructions for use 


\title{
Transmitting Pro-environmental Behaviors to the Next Generation: A Comparison between Germany and Japan
}

\begin{abstract}
The present study examined the processes in which children acquire pro-environmental behaviors in different cultures. We especially focused on the parental influence. Several studies have been conducted on adults' environmental behaviors, however we know little about how children's environmental attitudes and behaviors are formed.

We conducted a questionnaire survey with elementary school children and one of their parents in Germany and Japan. Number of pairs for the analysis was 221 in Germany and 365 in Japan. The results of structural equation modeling showed that parents' behaviors affected children's environmental behaviors directly and also via the subjective norm (the children's experienced expectations of their parents). A comparison of the two countries revealed that hypothesized cultural differences between the impact of personal norms and subjective norms were clearer for adults. The results also showed that the effects of subjective norms were stronger for children, indicating that children are more likely to be influenced by expectations of others. The results of the study suggest that for promoting children's environmental behaviors, showing the behaviors in daily life would be most effective.
\end{abstract}

Key words: children's environmental behavior, cultural comparison, observational learning, parental influence, paired analysis 


\title{
Transmitting Pro-environmental Behaviors to the Next Generation: A Comparison between Germany and Japan
}

\begin{abstract}
Introduction
Environmental issues, such as the increase of waste products, are globally seen as serious problems. Accordingly, adopting pro-environmental behavior can be considered social desirable in many cultures. A number of studies have investigated the determinants of pro-environmental behaviors (for a review, see Steg \& Vlek, 2009). However, these previous studies have, for the most part, conducted research that focuses on adult subjects; thus the processes through which we acquire pro-environmental norms and behaviors in childhood have not been fully investigated. We are not born with innate knowledge of environmental issues; rather, we must acquire attitudes, behaviors, and knowledge about the environment as we grow up.
\end{abstract}

This study investigates the processes by which children acquire pro-environmental behaviors. Uncovering these processes will contribute to the understanding of how the environmental behaviors of adults are formed. In this study we aim to compare environmental behaviors between children and adults.

Another issue addressed in the present study is whether there are cultural differences in parental influences on children, and if these have an effect on determinants of environmental behaviors. Environmentally-friendly behaviors can be seen globally but the reasons to adopt a particular behavior might be different across cultures. Understanding the cultural differences in determinants of environmental behaviors would be useful to further the understanding of the obstacles to environmentally friendly behaviors in different cultures. Currently 
there is almost no research investigating cultural differences of environmental behaviors in children, thus this research should provide a useful contribution to the field of environmental behavior studies.

In the present study we conducted a survey in which participants were elementary school children and their parents; specifically, we focused on one parent and one child in each household, and schools in two different countries, Germany and Japan, were chosen for the study. Therefore, this study has two axes of comparison: one is the comparison between children and adults, and the other is the comparison between two countries.

\section{Parental Influence on Children's Environmental Behavior}

For children, it can be assumed that parents would have a significant impact on their behavior, as parents are the primary facilitators of childhood socialization. Here, 'socialization' refers to the processes in which children acquire values, skills, and behavioral patterns that are considered as 'desirable' in the culture they live in (Brim, 1966). Parents are seen as the most important others for children until the higher grades in elementary school (Bower, 1979). While other external influences (e.g. media, peers) may account for discontinuities and inconsistencies, family environment has continuity and consistency (Grønhøj \& Thøgersen, 2009).

Parents may try to influence their children to adopt pro-environmental behaviors through verbal reinforcement, such as praising and warning, but according to social learning theory (Bandura, 1977), children primarily learn socially desirable values through observing behaviors of a model. Rogoff et al. (2003) pointed out that young children usually learn through observation without any reinforcement. Acquisition of language is an example of this type of learning (Akhtar et al., 2001). It has also been demonstrated in various studies that young children monitor events around them, and learn through observation (e.g. Piaget, 
1962; Trevarthen, 1977; Yando et al., 1989). We therefore predict that the environmental behavior of the parents should have significant effects on their children’s environmental behaviors.

In the domain of pro-environmental behaviors, several studies tested parental influences on children's environmental behaviors. Grønhøj and Thøgersen (2009, 2012) compared young adolescents' environmental values and behaviors with those of their parents, and found positive correlations in environmental attitudes and behaviors between young adolescents and their parents. They also reported young adolescents are environmentally less committed than their parents. Nakamura (2003) surveyed children (aged junior high school to young adolescents) - mother dyads on 13 pro-environmental behaviors. She found that the mothers' pro-environmental behaviors had a significant effect on that of their children, especially when the mothers requested their children to behave in specific ways. However, the mothers' behavior did not affect the children's environmental attitudes. Both Grønhøj and Thøgersen (2009, 2012) and Nakamura (2003)’s study demonstrated the linkage between parents' and children's environmental behaviors.

However, there have been very few studies focusing on elementary school age children. Grønhøj and Thøgersen (2009, 2012) and Nakamura (2003) surveyed young adolescents or those who were of high school age. For young adolescents, it is highly conceivable that their environmental attitudes and norms are already established and similar to those of adults. In order to understand the processes by which we acquire environmental norms at an early age research with younger children is necessary.

One study that focused on younger children is that of Evans et al. (2007) which examined elementary school first and second graders. The results did not show a significant relationship between children's pro-environmental behaviors 
and that of their parents. Yorifuji $(2003,2011)$ carried out research on children in grade fourth to sixth and one of their parents. In Yorifuji’s studies parents' behavior affected children's environmental behavior. She also showed that the effect of parental behavior was stronger than that of verbal reinforcement. There are difficulties in conducting research with younger children, and this may be the reason that Evans et al. (2007) did not find a relationship between children's behavior and parents' behavior.

Thus the present study aims to conduct a survey with elementary school third and fourth graders and one of their parents in order to understand the processes through which children acquire environmental norms and attitudes. We focused on children of this range of age, because age nine to ten was assumed to be the earliest age at which children can understand and answer the written questions. Since we used both children and parents as participants, the survey design allowed us to investigate the parental influence on children's behavior through paired analysis, not through parental behavior perceived by children.

In accordance with social learning theory (Bandura, 1977) and previous studies (e.g. Rogoff et al., 2003), we predict that parents' pro-environmental behaviors will affect children's pro-environmental behaviors.

\section{Cultural Differences}

A number of studies in cultural psychology have devoted attention to the dichotomy of individualism-collectivism (e.g., Triandis, 1988, 2001). In these studies, Germany is considered to have an individualistic culture, while Japan is said to have a collectivistic culture (Oetzel \& Ting-Toomey, 2003; Schimmack, Radhakrishnan, Oishi, \& Dzokoto, 2002). Similarly, Markus and Kitayama (1991) suggested that in Western cultures including Germany, an independent view of the self is dominant, while an interdependent view of the self is more dominant in 
Asian cultures including Japan. According to Markus and Kitayama (1991), fulfilling the expectations of others is more important in interdependent cultures, which implies that subjective norms, defined as expectations of significant others (Ajzen \& Fishbein, 1980; Fishbein \& Ajzen, 1975), would play a more important role in Japanese society. This notion is supported in Abrams, Ando, and Hinkle (1998), who found that subjective norms played an important role in workplace turnover in British and Japanese corporations. Ando, Ohnuma, and Chang (2007) and Ando et al. (2010) also found that subjective norms had a greater influence on environmental behavior in Japan when compared to the U.S. and Germany.

According to these studies, we can predict that subjective norms would play a more important role in determining the environmental behavior in Japan than in Germany, as greater importance is placed on interpersonal relationships in the Japanese culture. In Germany, it can be expected that personal norms would play a more important role because of the focus on individual values in Western cultures. This is supported by studies conducted in Europe showing that personal norms have a significant impact on environmental behaviors (e.g., Bamberg, Hunecke, \& Blöbaum, 2007; Hunecke, Blöbaum, Matthies, \& Höger, 2001; Matthies, Selge, \& Klöckner, 2010).

Milfont (2012) reviewed research showing that collectivistic individuals in a given country tend to be more environmentally concerned. However these studies were based on analyses conducted at an individual level and did not include the comparison between countries. Therefore it is not possible to predict that Japanese citizens would be more concerned about the environment than would their counterparts in Germany, and we do not have specific predictions about the mean level of environmental behavior in each country.

This is also the case with cultural differences in children's environmental 
behavior, as there is not much available evidence on the cultural differences in determinants of this behavior in children.

Children gradually learn cultural values during the process of socialization as they grow into adulthood. Thus it can be assumed that young children have not fully acquired cultural values, at least at the level that adults have. Therefore, we predict that cultural differences in determinants of environmental behaviors would be greater for adults than for children.

\section{Determinants of Environmental Behaviors in Children and Adults}

The present study explores the determinants of environmental behaviors in children and adults, with a focus specifically on the process of parental influence. It was assumed that parental behavior itself would directly affect children's behavior and would also have effects through subjective norms. In Yorifuji's (2003, 2011) study, it was found that parents' environmental behavior affects that of their children through subjective norms. Children perceived that parents expected them to adopt environmental behaviors when parents exhibited these behaviors themselves.

The subjective norm is defined as the perceived expectations from significant others and willingness to comply with them in the theory of reasoned action (TRA: Ajzen \& Fishbein, 1980; Fishbein \& Ajzen, 1975). The TRA proposes that behavioral intentions are determined by attitudes toward the behavior and also by subjective norms. The theory of planned behavior (TPB: Ajzen, 1985, 1991) expanded the TRA to incorporate perceived behavioral control as an additional direct determinant of behavior. The TPB has been employed in the research of pro-environmental behaviors in many countries (Ando et al., 2010; Groot \& Steg, 2007; Harland, Staats \& Wilke, 1999; Heath \& Gifford, 2002; Kaiser, et al., 2005; Stern et al., 1995; Nigbur, Lyons \& Uzzell, 2010; Taylor \& Todd, 1995; Yu-Long Chao, 2012). 
The present study measured subjective norms and perceived behavioral control as defined by TPB as a determinant of environmental behavior. We used personal norms, defined as "the feeling of personal moral obligation based on the individual's personal values” (Schwartz, 1977) Personal norm is a central variable in Schwartz's norm activation model (Schwartz, 1977; Schwartz \& Howard, 1980) and has been found to be a consistent predictor of environmental behavior (Bratt, 1999; Hunecke et al, 2001; Sterm, 2000; Widergren, 1998). In the norm activation model, as the first activation step, awareness of needs and responsibility are required as antecedents of personal norms (Schwartz, 1977). We incorporated personal norms in the analysis because they are more specific than general attitudes and also easier to differentiate as an internal determinant from the concept of a subjective norm, which is considered as an external determinant. The following analysis examines the relative importance of personal norms and subjective norms as internal and external values on the environmental behavior of the participants.

The predicted determinants for children's environmental behavior in the present study are shown in Fig. 1. Parents' behavior would affect children's behavior directly, as simple imitation of the observed behavior by children as suggested by Rogoff et al. (2003), and also mediated through subjective norms as found in Yorifuji’s study (2003, 2011). Personal norms and Perceived behavioral control (PBC) should also affect children's behavior independently. As a predictor of personal norms, we measured the perception of seriousness, which is the equivalent of awareness of needs in the norm activation model. Paths from parental behavior for perceptions of seriousness and PBC are not confirmed in the previous studies, but we aim to examine the effects in the present analysis for exploratory reasons. Parental behavior might affect children's perceptions of seriousness because it will show there actually is a problem which has to be taken seriously. 
PBC might be also affected by parental behavior because it will show the way to recycle used paper.

For parental behaviors, we also assumed that personal norms, subjective norms, and PBC would all act to determine behavior (Fig.2). We sought to compare the relative importance of these variables on behavior between children and parents.

Insert Fig. 1 The predicted determinants of children's environmental behavior

Insert Fig. 2 The predicted determinants of parents' environmental behavior

\section{Hypotheses}

The present study first aims to investigate parental influences on children's environmental behaviors. Observation learning theory (Bandura, 1977) and Rogoff et al. (2003) suggest that children learn new behaviors mainly through observation. In this study we examine whether environmental behaviors are also acquired through observation of parental behavior.

The second aim of the study is to compare the impact of subjective norms and personal norms on environmental behaviors in Germany and Japan. Greater importance is placed on individual values in Germany, which is considered to have an individualistic culture, thus personal norms should play a larger role in Germany than in Japan. In Japan, which is considered to have a collectivistic culture, interpersonal relationships have greater importance. Thus subjective norms should play a larger role in Japan than in Germany.

Our third aim is to compare the determinants of environmental behavior in 
children and adults. Since children are in the process of socialization, it is assumed that children are more sensitive to parental expectations, and are more likely to be influenced by external values. Therefore subjective norms would have stronger effects on children.

Hypotheses for the present study are as follows.

1a) Environmental behaviors of parents would directly affect the behavior of children. 1b) Environmental behaviors of parents would also affect children's environmental behaviors through subjective norms.

2) In Germany, personal norms should have larger effects on behavior compared to Japan, while subjective norms should have larger effects on behavior in Japan than in Germany.

3) Between children and parents, subjective norms should have larger effects on behavior for children than for parents.

\section{Method}

\section{Respondents}

A questionnaire survey was conducted in elementary schools in Cologne, Germany and Nagoya, Japan. Fourth grade students in 10 primary schools in Cologne and third and fourth grade students in 5 primary schools in Nagoya were asked to participate in the survey ${ }^{1}$.

\section{Procedures}

In Cologne 10 elementary schools ${ }^{2}$ cooperated in the present survey. The schools where the number of immigrants is relatively few were chosen for the present study in Germany because of cross cultural comparison. Teachers distributed questionnaires to students, and the students filled out the questionnaires in the classrooms. Subsequently each student took both this questionnaire and a second questionnaire home and asked one parent to complete the second form. 
Participants were given pre-paid envelopes and the parents returned the children's questionnaires, along with their own, together to the researchers using the envelopes.

In Japan, 27 schools in Nagoya were randomly chosen from 2 districts, which were chosen from 16 districts in the city. We sent letters to the 27 elementary schools requesting participation in the survey; this was followed by confirmation via telephone. Five schools allowed us to visit their campuses, and all five agreed to participate in the survey. In each school, teachers handed questionnaires to students in the third and fourth grades. All the students in the target grade received the questionnaires. Students then handed a second questionnaire to one of their parents at home. We asked all participants to fill the questionnaires separately without discussing each other's answers. Both completed questionnaires were sealed together in envelopes provided by the researchers and collected at the schools.

In both Germany and Japan, we asked the parent who felt most responsible for the household duties - such as cooking and cleaning - to fill in the questionnaire.

\section{Responses}

In Germany 284 pairs of responses were collected from the 680 questionnaires distributed. Responses in which a child's or parent's questionnaire was missing, those with many missing values, or those completed by respondents who indicated a nationality other than German, were excluded from the analysis. After these criteria were applied, we obtained 221 pairs of valid answers (the valid response rate was $32.5 \%)$

In Japan we collected 397 pairs of answers from the 478 questionnaires distributed. The same criteria as used for questionnaires collected in Germany were 
applied; the number of valid questionnaires was 365 (valid response rate $76.4 \%$ ).

\section{Measures}

We composed the questionnaires first in Japanese and then translated them into German. The translated questionnaires were verified by German native speakers, then pre-tested in both countries and adjusted. All items were measured using a five-point scale (1=strongly disagree, 5=strongly agree) except for behavior. Behavior was measured by a five -point scale (1=never, 2=seldom, 3=sometimes, 4=often, 5=always).

Behaviors. Pro-environmental behaviors of children and parents were measured by two items: 'I assort used paper so that it can be taken to collection of recyclables': cB1 / pB1, 'I separate used paper': cB2 / pB2. This assorting behavior was chosen from the pre-study as a behavior which is possible for school children to conduct in both countries because: 1) there is a recycling system for used paper in both countries, 2) it is possible for children to conduct this action at home, and 3) it is not an action that people take in order to save money.

Personal norms. Personal norms were measured by two items asking about an individual's feeling of an obligation to separate used paper ('I feel I am obliged to keep used paper to bring it for collection of recyclables': cPN1 / pPN1, 'I feel I am obliged to the environment to separate used paper':cPN2 and pPN2).

Subjective norms. Subjective norms in the children's questionnaire were also measured by two items asking about parents’ expectations of children to separate used papers ('My parents want me to separate used paper': cSN1: 'My parents want me to assort paper to the used paper container': cSN2). For parents' questionnaires, subjective norms were measured by one item ('My family expects me to separate used paper’: pSN)

Perceived behavioral control. Perceived behavioral control (PBC) was 
assessed by one item ('It is difficult for me to assort paper to the used paper container': PBC).

The questionnaires for both children and parents contained items related to the above variables unless otherwise specified.

Seriousness. We asked children about their perception of the seriousness of waste problems by two items ('It is a big issue for the people of Nagoya/Köln that there is so much waste': SP1, 'It is a serious problem that there is so much waste': SP2).

\section{Results}

All three hypotheses were tested using structural equation modeling (SEM). Below are demographic data on the respondents, a comparison of the means, correlations that give an outline of the data, and the results of the SEM.

\section{Demographics of Respondents}

About half of the children were female in both countries (49.8\% in Germany, $51.7 \%$ in Japan). Most of the parents were females, with a slightly higher percentage of females in the Japanese sample (83.6\% in Germany, $98.3 \%$ in Japan: $\left.\chi^{2}(1)=44.6, p<.001\right)$. The mean age of children was similar but statistically significantly higher in Germany [9.6 years old in Germany, 9.4 years in Japan: $t$ $(579)=3.56, p<.001]$. Parents' age was also slightly higher in Germany [41.09 in Germany, 38.89 in Japan: $t(567)=5.60, p<.001]$.

\section{Reliability of Measures}

Reliability of each measure was checked by cronbach's alpha. For children's questionnaire, alpha for German and Japanese respondents were as follows respectively. Behavior: .66 and .62, subjective norm: .88 and 68, personal norm: .71 and .63, seriousness: .71 and .64. For parents' questionnaire, behavior: .85 and .82, personal 
norm, .76 and .64. Alpha coefficients for each measure were not very high, but all the coefficients scored over .60, which showed reasonably high internal consistency.

\section{Cultural Differences in the Behavioral and Cognitive Variable Means}

Means of pro-environmental behavior and cognitive variables between two countries were compared by t-tests (Table 1). For the children's sample, behavior ( $t$ $(583)=5.22, p<.001)$, subjective norms $(t(584)=2.74, p<.01)$, and PBC $(t(584)=$ 8.43, $p<.001)$ were higher in Germany, while personal norms $(t(584)=-2.70, p<.01)$ were higher in Japan. As for the frequency of behaviors, $68.6 \%$ in Germany and $56.2 \%$ in Japan answered 'often' or 'always' $\left[x^{2}(2)=24.1, p<.001\right]$ for $\mathrm{cB} 1$. The corresponding number for cB2 was $64.1 \%$ in Germany and $43.1 \%$ in $\operatorname{Japan}\left[x^{2}(2)\right.$ $=14.0, p<.001]$. The largest difference was found for PBC, which showed Japanese children felt assorting paper was more difficult than did children in Germany. No significant difference was found for perceptions of seriousness, which were high in both countries.

In order to check the gender differences, $2 \times 2$ ANOVA using country and sex as independent variables were conducted for behavior and each cognitive variable. The result showed that main effect of sex and the interaction term of sex and country were not significant for all the variables except for seriousness $[F(1,565)=5.0, p<.05]$. The gender difference of seriousness was larger in Germany, in which male students scored higher than female students ( $M=4.44$ vs. 4.12 ). The main effects of country were significant for all the variables except for seriousness.

For the parent sample, behavior $(t(583)=12.59, p<.001)$, personal norms $(t$ $(584)=5.56, p<.001)$, subjective norms $(t(583)=2.10, p<.05)$, PBC $(t(583)=$

9.39, $p<.001$ ), were all higher in Germany. Especially, there were large differences for both behavior and PBC. German parents conducted assorting 
behavior more frequently than Japanese parents and perceived it to be an easier activity.

Insert Table 1 Means and standard deviation of behavioral and cognitive variables

\section{Correlations}

The correlations between the variables for children and parents are shown in Table 2. In Germany, children's behavior had significant correlations with all the variables. A relatively higher correlation was found with subjective norms ( $r=.632, p$ $<$.001). In Japan, the pattern of correlations with children's behavior and other variables were similar to that of the German sample, with a relatively higher correlation with subjective norms $(r=.520, p<.001)$. The correlations in the matrix ranged from low to medium, thus showing a low possibility of multicollinearity.

Insert Table 2 Correlations between children's and parents' behavioral and cognitive variables

\section{Determinants of Children's and Parents' Environmental Behaviors in Two \\ Countries}

Multi-group analysis was used to test the influence of parents' behavior on that of their children in Germany and Japan simultaneously. The analyses were conducted using AMOS 21. Full information maximum likelihood method was used for missing values.

Results for children's and parents' models are shown in Fig. 3. and Fig. 4. Model fit statistics indicated a good fit for both models (children's model: CMIN = 154.12, $d f=66, \mathrm{GFI}=.953, \mathrm{RMSEA}=.048$, parents’ model: $\mathrm{CMIN}=30.43, d f=$ 
10 , GFI $=.982$, RMSEA $=.059)^{3}$. The children's model explained $87 \%$ and $65 \%$ variation in children's environmental behavior in Germany and Japan respectively. The parents' model explained 65\% and 64\% variation in environmental behavior in Germany and Japan respectively. To compare the fit of the two countries, pairwise comparisons between the path coefficients were conducted. The significantly larger path coefficients are framed by rectangles.

Insert Fig. 3 The result of multi-group model between Germany and Japan for children's behavior

Insert Fig. 4 The result of multi-group model between Germany and Japan for parents’ behavior

Regarding hypotheses $1 \mathrm{a}$ and $1 \mathrm{~b}$ on the effects of parental behavior, results showed that, in both Germany and Japan, parental behavior had a direct effect on children's behavior (direct effect: .36 in Germany .19 in Japan) and also had an effect via subjective norms (indirect effect: .19 in Germany, .28 in Japan). The direct effect was significantly stronger in Germany. The significance levels of the indirect paths were tested by calculating confidence interval of the indirect effect ${ }^{4}$, which showed they were significant in both countries. Children perceived that they were expected to take assorting behavior by observing the behavior of their parents. Hypothesis 1a and 1b was supported in both Germany and Japan.

Parental behavior also affected the perception of seriousness, which determined personal norms. Personal norm affected children's behavior only in Germany. The indirect path from parents' behavior to children's behavior through seriousness and personal norm was significant (indirect effect: .06). In Japan the paths from parents' behavior to PBC, PBC to children's behavior were also significant, whose indirect effect was also marginally significant (indirect 
effect: .03).

As for the second hypothesis on cultural differences, the effect of personal norms was larger in Germany in the parents' model. The difference in path coefficient was significant. Only in Japan did subjective norms have a significant path coefficient on behavior in the parents' model. Therefore the results supported our hypothesis regarding cultural differences in the parents' model. For the children's model, the path from personal norms to the behavior was significant only in Germany. But the difference of the path coefficient was not significant. . For children, subjective norms had strong effects on behavior in both countries. Thus, the results showed that the cultural differences were larger for adults.

Regarding the third hypothesis on differences between children and parents, subjective norms had a significant impact on behavior for the children's model in both countries. The effect was much weaker for parents, although it was significant in Japan. On the other hand, the effect of personal norms was larger for parents. Children were more likely to be affected by the expectations of others. Thus, overall the results supported hypothesis 3.

\section{Discussion}

The present study examined parental influence on pro-environmental behavior of children through paired analysis of data collected from children and their parents. Regarding the parental influence on children's pro-environmental behavior, the results showed that parents' behavior affect children's behavior directly and through subjective norms. Learning through observation, as Rogoff et al. (2003) pointed out, was more influential in Germany, but it also had impact in Japan. Parental influence through subjective norm was found in both countries, which indicates the processes reported in Yorifuji's study (2003, 2011) are also common 
in Western countries.

The possibility that parents' behavior affect children's behavior via seriousness perception and PBC of children were tested in the present study exploratory. The results showed that parents' behavior could affect children's behavior by affecting seriousness perceptions and personal norm in Germany. By showing the behavior to children, parents are also showing that they consider the waste problems are serious matters which individuals need to cope with, which make children consider the issue as serious, that form children's personal norm. Parents' behavior also affected seriousness perception in Japan, but the link between personal norm and children's behavior was weaker. In Japan, parents’ behavior affected children's behavior through PBC. Parents' behavior showed how children can separate the used papers, where they can put the papers for recycling. The link between parents' behavior and PBC was not found in Germany; a possible reason may be that children in Germany already knew how to recycle paper.

The results indicate that parental influence on the behavior of their children is universal. The results of the present study support those shown in previous studies on children's environmental behaviors which found that parents are a powerful influence (e.g., Grønhøj \& Thøgersen, 2009, 2012; Nakamura, 2003; Yorifuji, 2003, 2011), which prove the consistency of Bandura's social learning theory (1977). The present study also showed that parents' behavior can also affect children's behavior through seriousness perception and PBC. The respondents of the present study were children of 9-10 years old, for whom parents would play a role as the most important others as Bower (1979) pointed out. The results indicated that in order to promote children's environmental behaviors, parents should be careful on their own behaviors, rather than trying to verbally change children's behavior.

Regarding the cultural differences, differences in the importance of personal 
norms and subjective norms were clearer for adults, this supported the hypothesis: personal norms were a more powerful determinant of the behavior in Germany, while subjective norms were significant only in Japan. This result was in line with previous findings that showed subjective norms had stronger effects in Japan (Abrams et al., 1988; Ando et al., 2007, 2010). It also suggests that since there is a greater importance placed on one's relationships with others in Asian cultures, the expectations of others play a larger role compared to their role in individualistic cultures. The cultural differences in the effect of subjective norm were not found for children's model. The results suggest that cultural differences are greater for adults as they have fully adapted to the cultural values of their respective societies, while children have not yet fully adapted to the cultural values.

One of the aims of the study was to compare the children and adults' model of environmental behavior. The SEM result showed that the effect of subjective norm is stronger for children. This indicated that children are more likely to be influenced by expectations of others. The results also showed that the personal norm effect was the strongest determinant of behavior for the adult model even in Japan, which indicates that personal values are relatively more important for adults. These findings may suggest that because children need to learn social expectations during the process of socialization, they are more sensitive to the expectations of others, while adults are more independent.

The results of cultural comparison and comparison of children and adults suggest: Subjective norms are internalized as a personal norm as we grow up, which may be a universal process. But there are cultural differences in the degree of internalization and the magnitude of the influence of subjective norms. We do not have enough evidence in the present findings to fully investigate this assumption, but it would worth further investigating this point as a topic of social psychology, not just in the 
area of environmental behavior.

\section{Mean Differences}

Comparison of the means revealed that the mean for assorting behavior was higher in Germany for both children and adults. Milfont (2010) argued that collectivistic individuals are more concerned with the environment; however it was not replicated in the present study which conducted country level comparison. Seriousness perception was relatively high in both Germany and Japan, which suggest that children of elementary school in both countries already have concerns about environmental issues.

There was a large cultural difference in the mean of PBC; it was higher in Germany for both children and adults. In Japan some of the respondents wrote in a space provided for free answers that they did not know paper is collected separately. If this is the case for some individuals in Japan, providing better information will promote assorting behavior.

\section{Conclusions}

The results of the present study suggested that considerable parts of the processes by which children acquire environmental behaviors are common, even in different cultures. Here specifically it was shown that children learn from the observation of parental behaviors.

The present study also suggested that as children grow up, they will adopt the cultural norms of the society in which they are raised, thus cultural differences in the determinants of the behaviors are larger for adults. This does also apply to the relative weight of the expectations from others and personal values.

The suggestions for promoting environmental behaviors from the present study would be that, for children, encouragement from others, especially demonstrating behavior in one's everyday life is effective because it enhances the cognition that this 
behavior is desired in a particular society. For adults, approaches to modify personal norms through communication such as demonstrating the seriousness of a problem are also seen as effective.

One of the major limitations of the study is the issue regarding measurements, which consisted of mainly one or two items. Since the present study aimed to gain responses from young children of ages 9-10, we tried to minimize the number of questions to get reliable answers, and the expressions were made simple. It was important to assure that children can understand the questions, but it would limit the statistical power of the analysis at the same time.

Another limitation of the present study is that it examined parental influence on children and did not incorporate other societal influences in its model. It has been pointed out in previous research that parental influence is one of the largest factors in a child's development. However it would be of interest to examine influences from other sources.

Despite some limitations, the present study offers a unique contribution in that we have analyzed paired data between children and adults, which enabled us to examine the effects of parental behavior on children's environmental behavior. We also compared the children's model between different cultures. Studies on children's environmental behaviors are few compared to those of adults; however this fact does not undermine the importance of studies on this area. Further research is necessary to illuminate the processes through which individuals acquire environmental attitudes and behaviors in a given society. 


\section{References}

Abrams, D., Ando, K. \& Hinkle S. (1998). Psychological attachment to the group: cross-cultural differences in organizational identification and subjective norms as predictors of workers' turnover intentions. Personality and Social Psychology Bulletin, 24, 1027-1039.

Ajzen, I. (1985). From intentions to action: A theory of planned behavior. In J. Kuhl \& J. Beckman. (Eds.), Action control: From cognitions to behaviors (pp. 11-39). New York: Springer.

Ajzen, I. (1991). The theory of planned behavior. Organizational Behavior and Human Decision Processes, 50, 179-211.

Ajzen, I. \& Fishbein, M. (1980). Understanding attitudes and predicting social behavior. Englewood Cliffs, NJ: Prentice Hall.

Akhtar, N., Jipson, J. \& Callanan, M. A. (2001). Learning words through overhearing. Child Development, 72, 416-430.

Ando, K., Ohnuma, S., Blobaum, A., Matthies, E. \& Sugiura, S. (2010). Determinants of individual and collective pro-environmental behaviors: comparing Germany and Japan. Journal of Environmental Information Science, 38 (5), 21-32.

Ando, K., Ohnuma, S. \& Chang, E. C. (2007). Comparing normative influences as determinants of environmentally conscious behaviours between the USA and Japan. Asian Journal of Social Psychology, 10, 171-178.

Bamberg, S., Hunecke, M. \& Blöbaum, A. (2007). Social context, personal norms and the use of public transportation: Two field studies. Journal of Environmental Psychology, 27, 190-203.

Bandura, A. (1977). Social Learning Theory. Englewood Cliffs, N.J: Prentice-Hall.

Bower, T. G. R. (1979). Human development. San Francisco, CA: W. H. Freeman.

Bratt, C. (1999). The impact of norms and assumed consequences on recycling behavior. 
Environment and Behavior, 31, 630-656.

Brim, O. (1966). Socialization through the life cycle. In O. Brim, \& S. Wheeler (Eds.), Socialization after childhood. New York: Wiley.

Evans, G. W., Brauchle, G., Haq, A., Stecker, R., Wong, K. \& Shapiro, E. (2007). Young children's environmental attitudes and behaviors. Environment and Behavior, 39, 635-658.

Fishbein, M. \& Ajzen, I. (1975). Belief, attitude, intention and behavior: An introduction to theory and research. Reading, MA: Addison-Wesley.

Grønhøj, A. \& Thøgersen, J. (2009). Like father, like son? Intergenerational transmissions of values, attitudes, and behaviors in the environmental domain. Journal of Environmental Psychology, 29 (4), 414-421.

Grønhøj, A. \& Thøgersen, J. (2012). Actions speak louder than words: The effect of personal attitudes and family norms on adolescents' pro environmental behaviour. Journal of Economic Psychology, 33 (1), 292-302.

Groot, J. D. \& Steg, L. (2007). General beliefs and the theory of planned behavior: The role of environmental concerns in the TPB. Journal of Applied Social Psychology, 37, 1817-1836.

Harland, P., Staats, H. \& Wilke, H. A. M. (1999). Explaining pro-environmental intention and behavior by personal norms and the theory of planned behavior. Journal of Applied Social Psychology, 29, 2505-2528.

Heath, Y. \& Gifford, R. (2002). Extending the theory of planned behavior: predicting the use of public transportation. Journal of Applied Social Psychology, 32, 21542185.

Hunecke, M., Blöbaum, A., Matthies, E. \& Höger, R. (2001). Responsibility and environment - Ecological norm orientation and external factors in the domain of travel mode choice behavior. Environment and Behavior, 33, 845-867. 
Kaiser, F. G., Hübner, G. \& Bogner, F. X. (2005). Contrasting the theory of planned behavior with the value-belief-norm model in explaining conservation behaviour. Journal of Applied Social Psychology, 35 (10), 2150-2170.

Markus, H. R. \& Kitayama, S. (1991). Culture and the self: Implications for cognition, emotion, and motivation. Psychological Review, 98, 224-253.

Matthies, E., Selge, S. \& Klöckner, C. A. (2010). Verantwortung für die Gesundheit von anderen? Eine Anwendung des Normaktivationsmodells auf nichtraucherschützendes Verhalten im universitären Kontext [Feeling responsible for the health of others? Applying a norm activation model to explain nonsmoker-protective smoking behaviour in a university setting]. Zeitschrift für Gesundheitspsychologie, 18 (3), 130-134.

Milfont, T. L. (2012). Cultural differences in environmental engagement. In S. Clayton (Ed.). Handbook of Environmental and Conservation Psychology. (pp. 181-202). Oxford: Oxford University Press.

Nakamura, M. (2003). Seinen no kankyou to kankyouhairyokoudou no keisei ni taisuru hahaoya no eikyou: gendou ikkannsei no kouka wo chushinni [Mothers' Influence on the development of their children's environmental consciousness and environment-conscious behavior]. Japanese Journal of Educational Psychology, 51, 76-85. (in Japanese)

Negayana, K. (1997). Oyakokankei to jiritsu In H. L. Kashiwagi, K., Kitayama, S., \& Azuma, H. (Eds.), Bunkashinrigaku [Cultural Psychology] (pp. 160-179). Tokyo: University of Tokyo Press.

Nigbur, D., Lyons, E. \& Uzzell, D. (2010). Attitudes, norms, identity and environmental behaviour: Using an expanded theory of planned behaviour to predict participation in a kerbside recycling programme. British Journal of Social Psychology, 49, 259-284. 
Oetzel, J.G. \& Ting-Toomey, S. (2003). Face concerns in interpersonal conflict: A cross-cultural empirical test of the face negotiation theory. Communication Research, 30, 559-624.

Piaget, J. (1962). Play, dreams, and imitation in childhood (translation by C.Gattengno\&F,M.Hodgson;original work published in 1951). New York: Noton.

Rogoff, B., Paradise, R., Mejía Arauz, R., Correa-Chávez, M. \& Angelillo, C. (2003). Firsthand learning through intent participation. Annual Review of Psychology, 54, 175-203.

Schimmack, U., Radhakrishnan, P., Oishi, S., Dzokoto, V. \& Ahadi, S. (2002). Culture, personality, and subjective well-being: integrating process models of life satisfaction. Journal of Personality and Social Psychology, 82, 582-593.

Schwartz, S. H. (1977). Normative influences on altruism. In L. Berkowitz (Ed.) Advances in Experimental Social Psychology. 10, 221-279. New York: Academic Press.

Schwartz, S. H. \& Howard, J. A. (1980). Explanations of the moderating effect of responsibility denial on the personal norm-behavior relationship. Social Psychology Quarterly, 43, 441-446.

Sobel, M. E. (1982). Asymptotic confidence intervals for indirect effects in structural equation models. Sociological Methodology, 13, 290-312.

Sobel, M. E. (1986). Some new results on indirect effects and their standard errors in covariance structural models. Sociological Methodology, 16, 159-186.

Steg, L. \& Vlek, C. (2009). Encouraging pro-environmental behavior: An integrative review and research Agenda. Journal of Environmental Psychology, 29, 309-317

Stern, P. C., Kalof, L., Dietz, T. \& Guagnano, G. A. (1995). Values, beliefs and pro-environmental action: Attitude formation toward emergent attitude objects. Journal of Applied Social Psychology, 25, 1611-1636. 
Taylor, S. \& Todd, P. (1995). An integrated model of waste management behavior: a test of household recycling and composting interaction. Environment and Behavior. 27, 603-630.

Trevarthen, C. (1997). Fetal and neonatal psychology: Intrinsic motives and learning behavior. In F. Cockburn (Ed.), Advances in Perinatal Medicine, (pp. 282-291). (Proceedings of the XVth European Congress of Perinatal Medicine, Glasgow, 10-13 September, 1996) .

Triandis, H. C., Bontempo, R., Villareal, M., Asai, M. \& Lucca, N. (1988). Individualism and collectivism: Cross-cultural perspectives on self-in-group relationships. Journal of Personality and Social Psychology, 54,323-338.

Triandis, H. C. (2001). Individualism-Collectivism and Personality. Journal of Personality, 69, 907-924.

Yando, R., Seitz, V. \& Zigler, E. (1989). Imitation, recall, and imitativeness in children with low intelligence of organic and familial etiology. Research in Developmental Disabilities, 10(4), 383-397.

Yorifuji, K. (2003). Kodomo no gomi genryoukoudou ni oyobosu syakaiteki eikyou. [Social influence by parents on children's waste reduction behavior]. Journal of the Japan Society of Waste Management Experts, 14, 166-175. (in Japanese)

Yorifuji, K. (2011). Kodomo no gomi genryoukoudou no kitei-in toshiteno kojinkihan to shakaitekieikyou [Personal and social norms that determine children's waste reduction behavior]. The Japanese Journal of Psychology, 82 (3), 240-248. (in Japanese)

Widegren, Ö. (1998). The new environmental paradigm and personal norms. Environment and Behavior, 30, 75-100.

Yu-Long Chao (2012). Predicting people’s environmental behavior: theory of planned behavior and model of responsible environmental behavior. Environmental 
Education Research, 18 (4), 437-461. 


\section{Endnotes}

${ }^{1}$ We recruited third and fourth grade students in Japan because of differences in the school system in Germany and Japan. In Germany, children can start elementary school as early as five years old, while in Japan children begin elementary school at six.

${ }^{2}$ We conducted the survey at a larger number of schools in Germany, as student enrollment in each grade is smaller than in Japan.

3 The null hypothesis for the model was rejected, however Hoelter's critical $\mathrm{N}$ for 5 \% significance level was 353 for parents’ model and 327 for children’s model. The number of the pairs used for the analysis was 586, which exceeds both values; this indicates that the model was rejected because the sample size was large.

${ }^{4}$ Confidence interval of the indirect effect was calculated based on standard errors, which was calculated by delta method (Sobel, 1982, 1986). It was considered as significant when the 95\% confidence interval of the indirect effect does not include zero. 
Figure legends

Table 1 Means and standard deviation of behavioral and cognitive variables

Table 2 Correlations between children's and parents' behavioral and cognitive variables

Figure 1 The predicted determinants of children's environmental behavior

Figure 2 The predicted determinants of parents' environmental behavior

Figure 3 The result of multi-group model between Germany and Japan for children's behavior

Figure 4 The result of multi-group model between Germany and Japan for parents' behavior 
Table 1

Means and standard deviation of behavioral and cognitive variables

\begin{tabular}{|c|c|c|c|c|}
\hline & \multicolumn{4}{|c|}{ Country } \\
\hline & Germany & Japan & Germany & Japan \\
\hline & \multicolumn{2}{|c|}{ Children } & \multicolumn{2}{|c|}{ Parents } \\
\hline Behavior & 3.77 (1.19) & 3.22 (1.24) & 3.87 ( . .75) & 2.53 (1.27) \\
\hline Personal norm & 3.79 (1.05) & $4.02(.97)$ & 4.36 ( . .79) & 3.94 ( .93) \\
\hline Subjective norm & 3.89 (1.20) & 3.62 (1.10) & 3.43 (1.37) & $3.20(1.15)$ \\
\hline PBC & $4.00(1.21)$ & 3.08 (1.32) & 4.42 ( .95) & 3.49 (1.27) \\
\hline Seriousness & $4.28(.85)$ & $4.16(.92)$ & - & - \\
\hline
\end{tabular}

$(S D)$ 
Table 2

Correlations between children’s and parents' behavioral and cognitive variables

\begin{tabular}{|c|c|c|c|c|c|c|c|c|c|c|c|c|}
\hline & & 1 & 2 & 3 & 4 & 5 & 6 & 7 & 8 & 9 & 10 & 11 \\
\hline \multirow{4}{*}{ Children } & 1. Behavior & - & $.496^{* * *}$ & $.632^{* * *}$ & $.419^{* * *}$ & $.412^{* * *}$ & $.444^{* * *}$ & $.362^{* * *}$ & $.239^{* * *}$ & $.188^{* *}$ & $.375^{* * *}$ & $.216^{* * *}$ \\
\hline & $\begin{array}{l}\text { 2. Personal } \\
\text { norm }\end{array}$ & $.409^{* * *}$ & - & $.539^{* * *}$ & $.227^{* * *}$ & $.433^{* * *}$ & $.213^{* * *}$ & $.194^{* *}$ & $.228^{* * *}$ & .058 & $.319^{* * *}$ & $.224^{* * *}$ \\
\hline & $\begin{array}{l}\text { 3. Subjective } \\
\text { norm }\end{array}$ & $.520^{* * *}$ & $.488^{* * *}$ & - & $.307^{* * *}$ & $349^{* * *}$ & $.386^{* * *}$ & $.315^{* * *}$ & $.282^{* * *}$ & $.231^{* * *}$ & $.393^{* * *}$ & $.233^{* * *}$ \\
\hline & 4. $\mathrm{PBC}$ & $.243^{* * *}$ & $.145^{* *}$ & $.195^{* * *}$ & - & $.179^{* *}$ & .087 & -.050 & -.047 & .041 & .041 & -.006 \\
\hline \multirow{4}{*}{ Parents } & 6. Behavior & $.416^{* * *}$ & $.251^{* * *}$ & $.464^{* * *}$ & $.172^{* * *}$ & $.138^{* *}$ & - & $.639^{* * *}$ & $.360^{* * *}$ & $.322^{* * *}$ & $.525^{* * *}$ & $.305^{* * *}$ \\
\hline & $\begin{array}{l}\text { 7. Personal } \\
\text { norm }\end{array}$ & $.287^{* * *}$ & $.192^{* * *}$ & $.348^{* * *}$ & $.134^{*}$ & $.170^{* * *}$ & $.570^{* * *}$ & - & $.494^{* * *}$ & $.261^{* * *}$ & $.619^{* * *}$ & $.479^{* * *}$ \\
\hline & $\begin{array}{l}\text { 8. Subjective } \\
\text { norm }\end{array}$ & $.297^{* * *}$ & $.213^{* * *}$ & $.286^{* * *}$ & $.135^{* *}$ & $.111^{*}$ & $.505^{* * *}$ & $.526^{* * *}$ & - & $.185^{* *}$ & $.411^{* * *}$ & $.346^{* * *}$ \\
\hline & 9. $\mathrm{PBC}$ & $.251^{* * *}$ & $.195^{* * *}$ & $.321^{* * *}$ & .083 & $.108^{*}$ & $.552^{* * *}$ & $.455^{* * *}$ & $.327^{* * *}$ & - & $.193^{* *}$ & $.139^{*}$ \\
\hline
\end{tabular}

Note. 1 Correlations for German sample are above the diagonal; those for the Japanese sample are below the diagonal $2 * * * p<.001, * * p<.01, * p<.05$. 


\section{Figure1}

The predicted determinants of children's environmental behavior

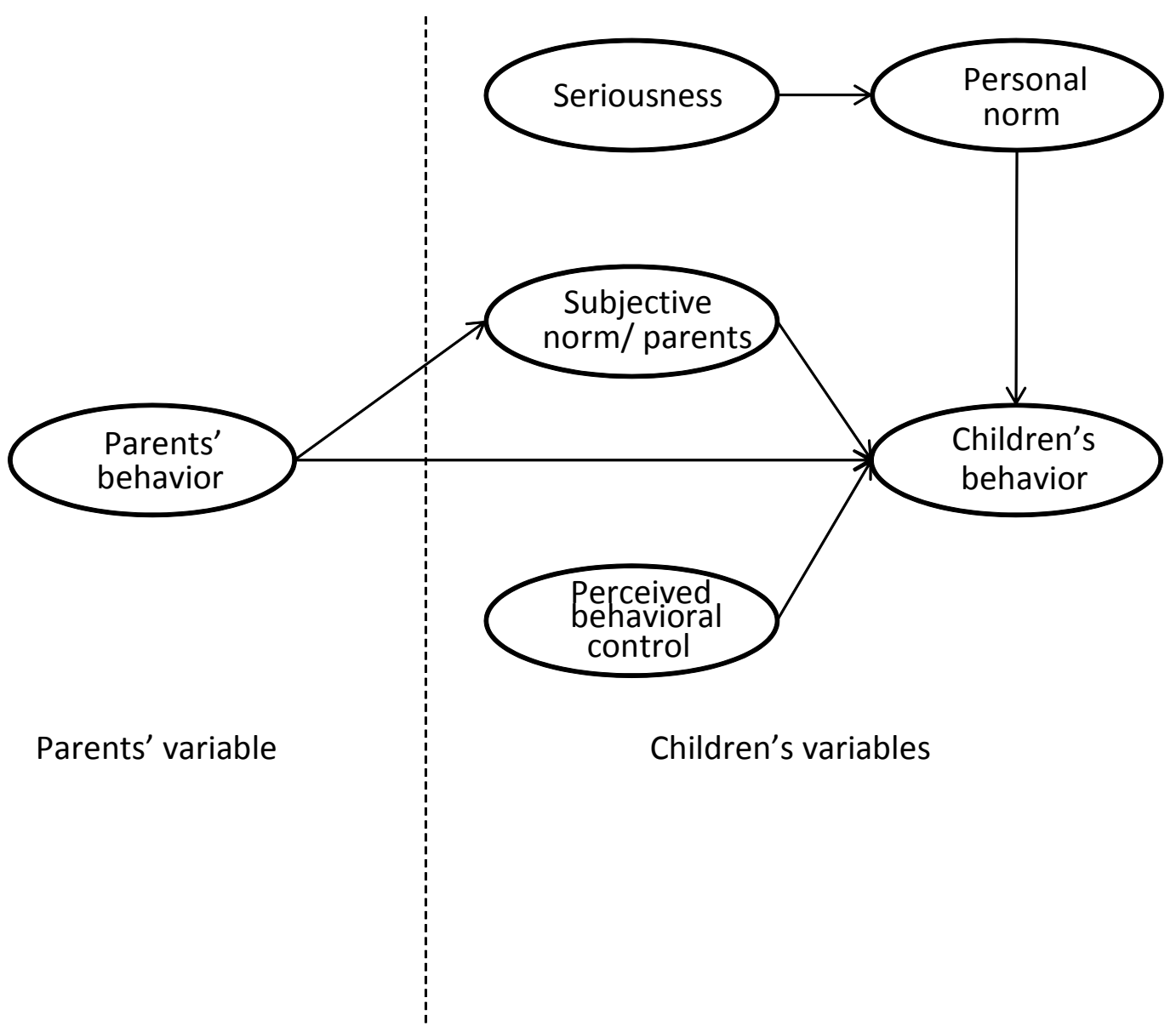


Figure 2

The predicted determinants of parents' environmental behavior

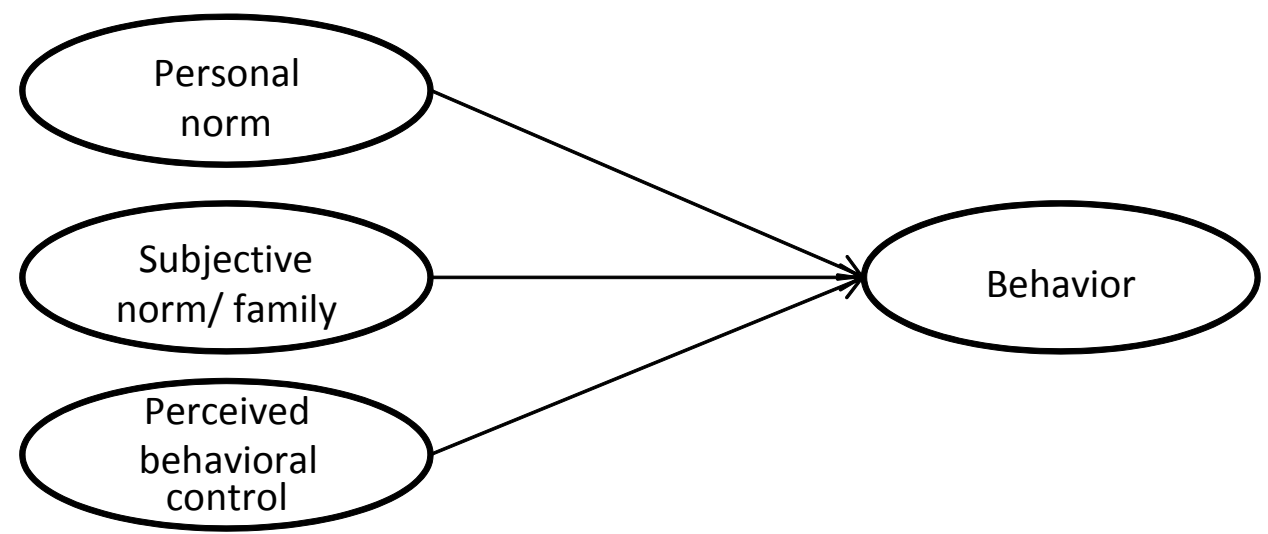


Figure 3

The result of multi-group model between Germany and Japan for children’s behavior

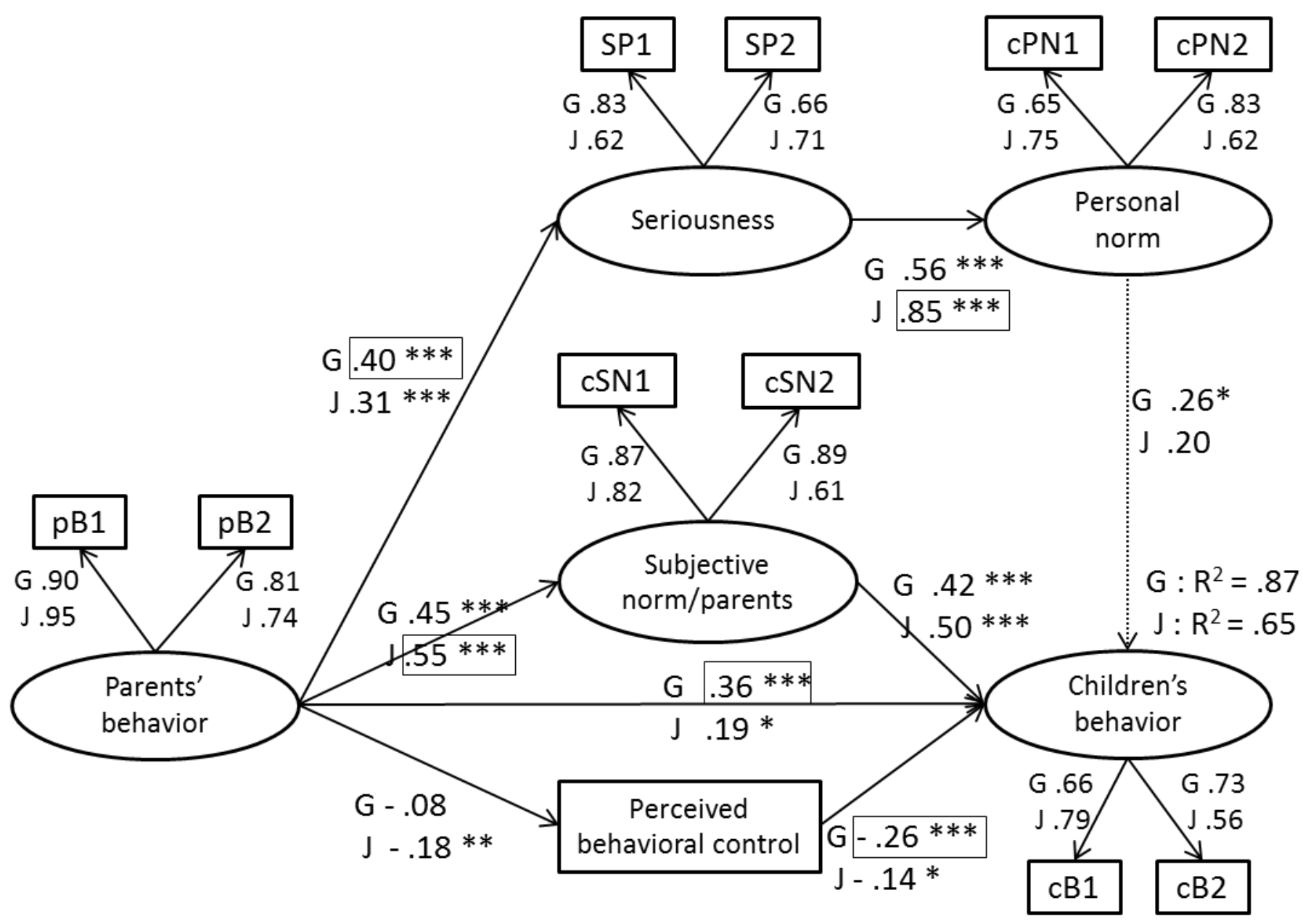

Note.

$1 * * * p<.001, * * p<.01, * p<.05$.

2 Country: $\mathrm{G}=$ Germany, $\mathrm{J}=$ Japan.

3 Model fit: $\chi^{2}(66)=154.12$, GFI $=.953$, AGFI $=.906$, CFI $=.958$, RMSEA $=.048$

4 The significantly larger path coefficients are framed by rectangles. 
Figure 4

The result of multi-group model between Germany and Japan for parents’ behavior

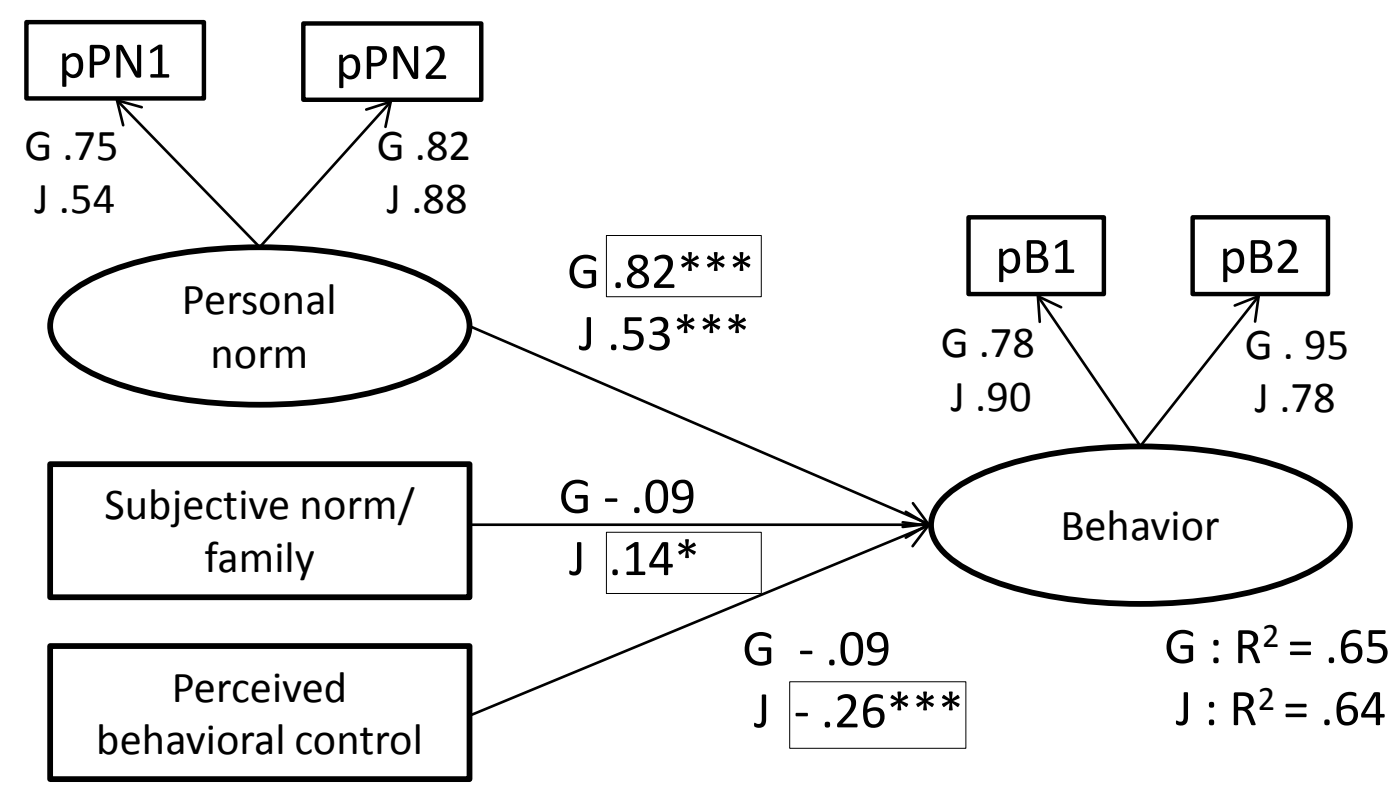

Note.

$1 * p<.001, * * p<.01, * p<.05$.

2 Country: $\mathrm{G}=$ Germany, $\mathrm{J}=$ Japan.

3 Model fit: $\chi^{2}(10)=30.43$, GFI $=.982$, AGFI $=.924$, CFI $=.984$, RMSEA $=.059$

4 The significantly larger path coefficients are framed by rectangles. 